\title{
Aircraft Mission Simulation Framework for Loads Analysis
}

\author{
Simon Schulz *, Daniel Ossmann ${ }^{\dagger}$, Daniel Milz ${ }^{\ddagger}$, Thiemo Kier ${ }^{\S}$, and Gertjan Looye ${ }^{\llbracket l}$ \\ Institute of System Dynamics \& Control \\ German Aerospace Center (DLR), 82234 Wessling, Germany
}

\begin{abstract}
The analysis of structural usage data of aircraft becomes increasingly relevant in today's aircraft industry to optimize operational efficiency. As this data is not always available due to the lack of load sensors, a framework is proposed herein which allows to re-simulate real flights and estimate the encountered loads. The framework's core element is a flexible aircraft simulator consisting of flexible, open-loop aircraft dynamics together with a flight control system (FCS). Thereby, closed-loop simulation capabilities to simulate flight missions in auto-pilot mode are provided. To re-simulate real flights, an input data handling module processes real timelines of ground speed and GPS coordinates to generate a mission profile, which provides the necessary inputs to the FCS. The simulated loads allow detailed analyses of the structural usage over user-defined time spans, varying from single flights to whole aircraft lifetimes. As example, the structural usage of a small fleet over two years in scheduled service is analyzed. To increase computational efficiency, the flown routes in reality are approximated by a representative set.
\end{abstract}

\section{Introduction}

To fulfill the greener imperative demanded by society, various approaches are taken by the aircraft industry today to improve the environmental footprint of commercial aircraft. This includes more efficient engines, structural weight reductions, better aerodynamic characteristics of wings and control surfaces [1], and even active control techniques to reduce encountered loads [2, 3]. All these aspects by themselves are main drivers for decreasing fuel and cost consumption. A common aspect which drives the design of aircraft in general are the encountered loads during flight. On the one hand, these loads define the structural design envelope and thereby directly influence the fuel consumption of the aircraft when in service. On the other hand they also have a main impact on the maintenance schedule and thereby on operational costs and usage of replacement parts as they define the predefined service intervals. As consequence, a better understanding of the fatigue loads an aircraft encounters over its whole life time could help to reduce design conservatism. This knowledge can be feed back into the structural design envelope as well as in the definition of service intervals in the future.

In more detail, one specific fatigue driven topic, which accounts for a large percentage of direct operation cost, is so-called Maintenance Repair and Overhaul (MRO). Currently, aircraft inspections are planned in predefined rigid intervals. Continuously monitoring the health of the aircraft structure would enable a more dynamic maintenance planning based on the condition of the components. The usage based planning of maintenance is also called Condition based maintenance (CBM) [4]. Within the EU-Horizon 2020 Project DEMETER a CBM approach for the engine pylon is proposed. First, loads at the engine center of gravity are estimated using recorded operational mission data together with a model based approach. The loads are used to estimate stresses in the specific component. Within the fatigue analysis loads are then used to determine a health index, which allows deriving a dynamic maintenance plan.

The discussions above reveal, however, that there exists an inevitably demand of either measuring or estimating the encountered loads over entire mission profiles. Measuring these loads would be too cost sensitive so that the estimation is the more realistic way to pursue. At present, load simulations are performed to investigate single events, i.e. a very small subset of the actual events encountered by the aircraft. Typically, maneuvers like $2.5 \mathrm{~g}$ pull up or atmospheric disturbance encounters like discrete gusts are simulated to define the structural load envelope during design, possibly introducing a huge conservatism in the aircraft design. Thus, to provide an increased understanding of fatigue loads

\footnotetext{
*Research Associate: Simon.Schulz@dlr.de.

$\dagger$ Professor at HM Munich, former DLR: Daniel.Ossmann@hm.edu, AIAA Senior Member.

†rainee: Daniel.Milz@dlr.de.

§esearch Scientist: Thiemo.Kier@dlr.de, AIAA Member.

IDepartment Head: Gertjan.Looye@dlr.de.
} 
over a whole life cycle, this paper tackles the need for a-posteriori load calculations for whole aircraft missions and proposes a simulation based framework in this paper.

Within this paper, the framework for loads estimation based on operational data from real world flight missions is introduced. The structure of the proposed framework is described in detail in Section II. This includes the mission database and the mission selection as well as the simulation environment on the inputs side, the aircraft simulation model as core element of the framework, and finally, the load estimation to provide the framework's outputs. A detailed discussion on the closed loop flight and structural dynamics aircraft model is provided. The functionality of the framework is demonstrated on a selection of flight missions in Section III.

\section{Loads Estimation Framework}

The purpose of the framework is to simulate real flight missions and provide an estimate of the encountered loads during these missions. The framework dedicated to this task is displayed in Fig. 1. The Mission Database includes all

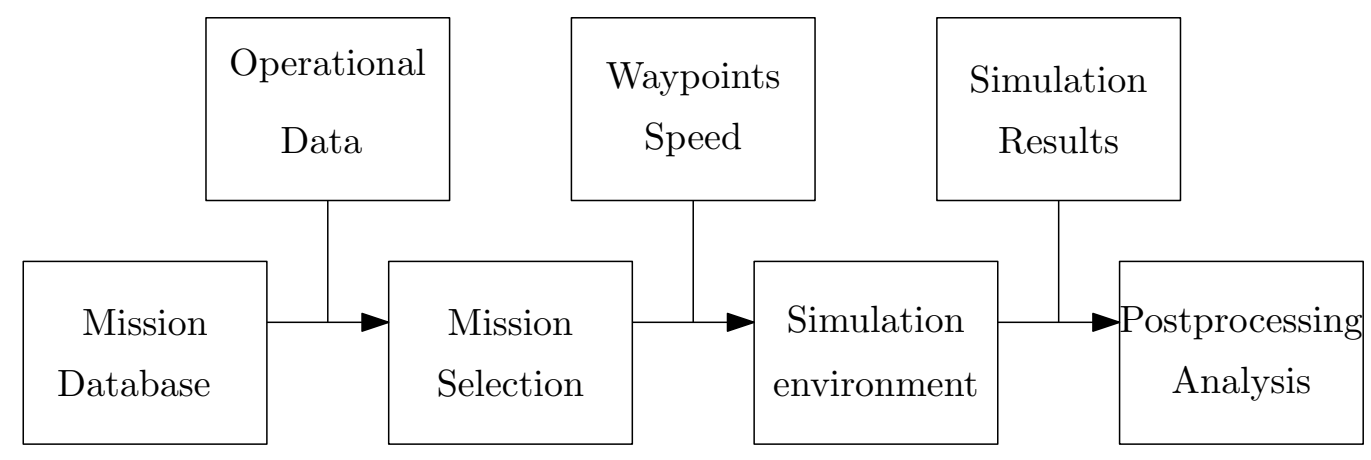

Fig. 1 Multiple flight trajectories.

data about the available flight missions. In order to keep the number of missions in a scale, which can be processed by a standard desktop computer, representative missions need to be determined. The "Mission selection" block offers this functionality. In detail, the operational data contains the waypoints of the flown trajectories during the missions, as well as the flown ground speeds. Based on these data, the simulation environment then calculates the required flight parameters for the load estimation, which is conducted in the "Postprocessing Analysis" block. Instead of directly calculating the loads in the simulation model, it is compelling to estimate the loads in a specific post-processing step. Otherwise the produced loads data during the simulation would exceed the memory capacity of a desktop computer. Additionally, this reduces the overall computation time.

\section{A. Mission Database and Mission Selection}

The mission data base contains the data available from real flight missions. The data is separated into two levels of detail for each flight mission. The first, more general, level of detail contains the following information:

- Departure Time

- Departure airport
- Arrival Time

- Arrival airport
- Aircraft type

- Flight time

Within "Missions Selection", the mission information is processed in order to choose the missions the loads will be estimated for. Constraints regarding number of missions arise from simulation time as well as amount of data generated during the simulation process.

The second level of detail contains specific information about the flight trajectory. The trajectory is discretized by waypoints, which are characterized by longitude, latitude and altitude. Additionally, the corresponding groundspeed profile is included. A typical representation of a trajectory discretized by waypoints is displayed in Fig. $2 \mathrm{a}$ and Fig. $2 \mathrm{~b}$ The corresponding groundspeed profile is shown in Fig. $2 \mathrm{c}$. 


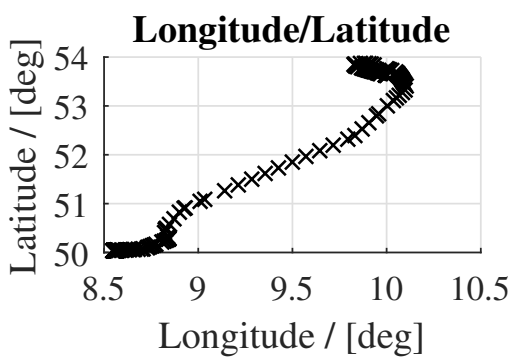

(a) Longitude/Latitude coordinates

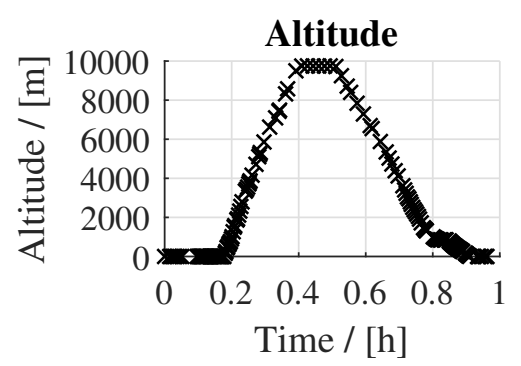

(b) Altitude

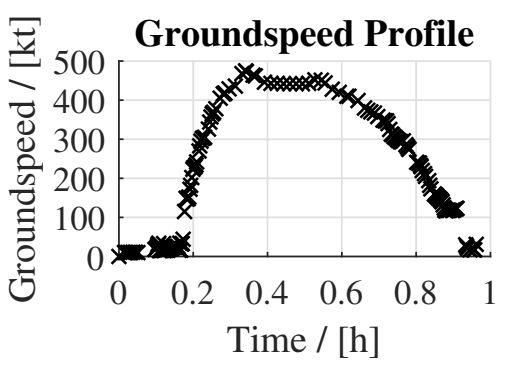

(c) Groundspeed

Fig. 2 Operational input data illustration.

\section{B. Simulation Environment and Loads Estimation}

The simulation environment is able to describe the flight and structural dynamics of an aircraft on trajectories, defined in the previous section, and derive the loads during these missions. Core of the simulation environment is a flight and structural dynamics physical aircraft model. The model is derived using the framework "Varloads" [5][6]. Originally, this framework is designed to offer the opportunity to investigate single events in a timeframe of about 10 seconds. The model provided is an integrated flight loads model. The structural model within the flight loads model is based on a "beam-like" structural model. The stiffness matrix $K_{g g}$ of a beam-like structural model can be derived from a finite element model by a reduction method like the Guyan reduction with condensation points (g-set) along the loads reference axis [5]. The condensation points represent the nodes of the beam-like model. The mass properties $M_{g g}$ of the beam-like aircraft structure are described by lumped masses, which are attached to the nodes of the beam-like model. For the structural model, a modal analysis is conducted. The modal basis is reduced in order to reduce the degrees of freedom. The modal degrees of freedom are divided in the rigid-body states (b-set) and the flexible states (f-set). The modal basis for the rigid body states is given in $\phi_{g b}$, and for the flexible states in $\phi_{g f}$. Based on that, the nonlinear equations of motion of the flight and structural dynamics model, which describes the aircraft movement relative to "mean axis" body reference frame is derived. The equations are defined by

$$
\left[\begin{array}{c}
m_{b}\left(\dot{V}_{b}+\Omega_{b} \times V_{b}-T_{b E} g_{E}\right) \\
\dot{J}_{b} \dot{\Omega}_{b}+\Omega_{b} \times\left(J_{b} \Omega_{b}\right)
\end{array}\right]=\Phi_{g b}^{T} P_{g}^{\text {ext }}(t) .
$$

Inertia properties are modeled by a point mass $m_{b}$ and the corresponding rotational intertia $J_{b}$. At the current state of the framework, only one mass configuration is taken into account for the simulation of a whole mission. $V_{b}$ is the velocity, $\Omega_{b}$ the angular velocities in the body fixed frame (b-set). $T_{b E}$ transforms the gravitational vector from an earth fixed coordinate system into the body-fixed frame of reference, indicated with the index $b$. The elastic dynamics are described by the linear second order differential equations Eq. (2).

$$
M_{f f} \ddot{u}_{f}+B_{f f} \dot{u}_{f}+K_{f f} u_{f}=\Phi_{g f}^{T} P_{g}^{\mathrm{ext}}(t)
$$

$M_{f f}$ is the mass matrix, $B_{f f}$ the damping matrix and $K_{f f}$ the stiffness matrix. $P_{g}^{\text {ext }}$ are the external loads. They consist of the aerodynamic loads $P_{g}^{\text {aero }}$ and propulsion forces $P_{g}^{\text {prop }}$, i.e.

$$
P_{g}^{\text {ext }}=P_{g}^{\text {aero }}+P_{g}^{\text {prop }}
$$

The calculation of the aerodynamic loads $P_{g}^{\text {aero }}$ is based on the vortex lattice method Aerodynamic Influence Coefficient (AIC) and given by

$$
P_{g}^{\text {aero }}=q_{\infty} T^{T} S Q w,
$$

where $q_{\infty}$ describes the dynamic pressure. $T$ is the splining matrix, which couples the aerodynamic model with the structural model, $S$ sums up the pressure difference at each panel, and $Q$ is the Aerodynamic Influence Coefficient(AIC) matrix. The normalized wind velocity, acting at each aerodynamic panel normal to the surface, is descibed by $w$. The calculation of the aerodynamic loads is based on a linearized potential flow approximation. For more details see [5]. The aerodynamic model also provides the loads on the airframe due to the control surfaces, elevator, ailerons and rudder. The propulsion forces $P_{g}^{\text {prop }}$ act at the engine nodes of the structural model. In order to derive the loads 
during a simulated mission, the so called load recovery is done according to [5], where the force summation method is applied. The idea of force summation is to add up all loads acting on the airframe to derive the internal structural loads. Therefore the inertia $P_{g}^{\text {iner }}$, aerodynamic $P_{g}^{\text {aero }}$ and propulsion forces $P_{g}^{\text {prop }}$ are summed up. To derive the nodal loads $P_{g}$ acting on the structural grid the load recovery equation is defined with Eq. (3) as

$$
P_{g}=P_{g}^{\text {ext }}-P_{g}^{\text {iner }}=P_{g}^{\text {aero }}+P_{g}^{\text {prop }}-P_{g}^{\text {iner }}
$$

The aerodynamic loads $P_{g}^{\text {aero }}$ are given by Eq. 4 . The loads $P_{g}^{\text {iner }}$ due to inertia, depending on the modal accelerations $\ddot{u}_{f}$ (Eq. (2)) and $\ddot{u}_{b}$, can be derived from Eq. (1) as

$$
P_{g}^{\text {iner }}=M_{g g}\left\{\Phi_{g f} \ddot{u}_{f}+\Phi_{g b} \ddot{u}_{b}\right\}
$$

with the rigid body acceleration

$$
\ddot{u}_{b}=\left[\begin{array}{l}
\left(\dot{V}_{b}+\Omega_{b} \times V_{b}-T_{b E} g_{E}\right) \\
\dot{\Omega}_{b}+J_{b}^{-1}\left(\Omega_{b} \times\left(J_{b} \Omega_{b}\right)\right)
\end{array}\right] .
$$

\section{Flight Control System}

Target of the simulation environment is to estimate the encountered loads on the flown trajectories, which are available by discretized by waypoints. Eq. (5) determines the loads depending on the flight and structural dynamics. Therefore, a function is required, which keeps the flight and structural dynamics model of the aircraft on defined trajectories. For this purpose, an electronic flight control system (ECFS) is included in the simulation environment. The whole simulation environment is displayed in detail in Fig. 3 .

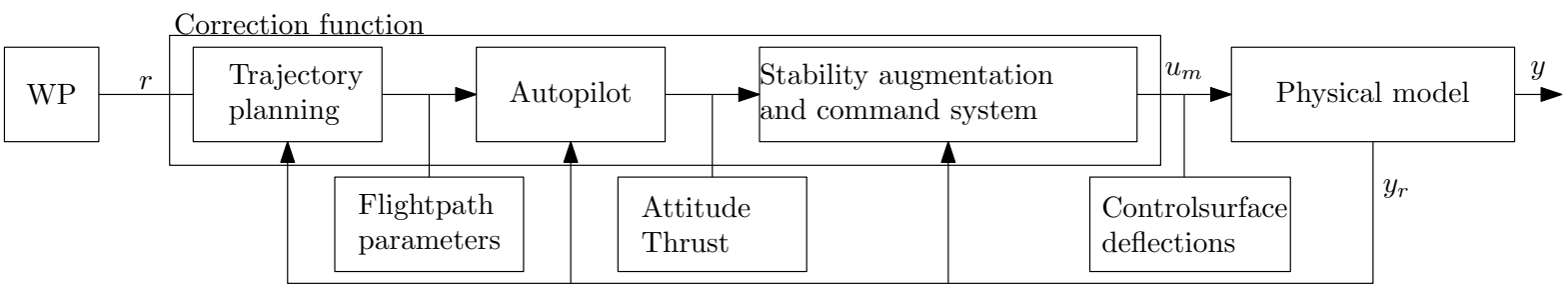

Fig. 3 Block diagram of simulation environment.

Within the environment, the waypoints (provided by the mission selection, see Fig. 1), are translated into control surface commands in order to keep the flight and structural dynamics model on the trajectory defined by the waypoints. The block which manipulates the physical model according to the waypoints is called "correction function". The structure of the correction function is similar to an ECFS. The EFCS consists of a trajectory planning block, autopilot control law as well as stability augmentation and command system

The simulation environment, shown in Fig. 3, consists of a guidance navigation and control system (GNC) as well as a physical model of the aircraft. similarly to the EFCS, GNC-system consists of a trajectory planning block, an autopilot and a stability augmentation and command system block. The GNC system block has the task to keep the physical model of the aircraft on the trajectory, defined by the waypoints. This task is split into three sub-tasks for the GNC-system. The first task, handled by the trajectory planning block, is to derive the trajectory from the waypoints and command the flightpath parameters $\left(\chi_{\mathrm{com}}, \gamma_{\mathrm{com}}, h_{\mathrm{com}}\right)$, taking into account the current position of the aircraft with respect to the trajectory to keep the aircraft on the trajectory. The flightpath parameters are the input for the autopilot, which translates the flightpath parameters commands into attitude commands. The attitude commands consist of roll and pitch angle commande, i.e., $\phi_{\text {com }}$ and $\theta_{\text {com }}$, as well as the side slip angle command $\beta_{\text {com }}$ commands. Furthermore, the autopilot commands the thrust. The autoflight-flight-control-laws are based on total energy control systems (TECS) [7] and total heading control systems THCS [8]. The stability and command augmentation system processes the attitude commands of the autopilot and translates them into control surface deflections, which manipulate the aircraft. The stability augmentation and command system is based on the incremental non-linear dynamic inversion (INDI) methodology explained in [9].

Note that the framework is set up in modular manner. Thus, if the actual flight control system of the aircraft is available, this can be easily taken into account by replacing the included FCS functions with the new one. However, as this is not often the case due to propriety reasons, a generic FCS is provided which allows to re-simulate the flown trajectories and estimate the encountered loads. 


\section{Application of the Load Estimation Framework}

For demonstration of the framework a fleet is investigated. The fleet $A C_{J}$ consists of $J=36$ aircraft $A C_{j}$. The goal is to approximate the loads during flight with available trajectory as well as ground data. The data is available for an interval of two years. During these two years $N^{A C_{j}}=134984$ missions were recorded. Due limited computational capacity, would be clearly exceeded by aeroelastic simulation of $N^{A C_{j}}$ mission-trajectories, representative missions need to be determined in order to find representative load-time histories for the fleet $A C_{J}$.

By investigating the mean mission duration (MMD) $\bar{T}^{A C_{j}}$ of the $j^{\text {th }}$ aircraft, the relation between the number of ground-air-ground cycles to in-flight time is given, assuming that every aircraft has the same operation time per day. To find a representative aircraft for the whole fleet, the MMD of the representative aircraft $\bar{T}^{A C_{j}}$ should be similar to the MMD of the fleet $\bar{T}^{A C_{J}}$. For the application example herein, the MMD is given by

$$
\bar{T}_{J}=\sum_{n=1}^{N} \frac{T_{n}}{N^{A C_{J}}}=1.424,
$$

where $T_{n}$ is the mission duration of the $n^{\text {th }}$ recorded mission. Thus, the MDD of the discussed fleet $\bar{T}_{J}$ is 1.424 hours. Similar to the fleet's MMD, $\bar{T}^{A C_{j}}$ describes the MMD of an individual aircraft $j$. The five aircraft with most similar MMD $\bar{T}^{A C_{j}}$ compared to the fleet MMD are documented in Table 1 . The difference in MMD between the fleet $A C_{J}$ and each individual aircraft is quantified by the absolute difference

$$
\left|\epsilon_{\bar{T}}\right|=\left|\bar{T}_{J}-\bar{T}_{j}\right| .
$$

\begin{tabular}{|c|c|c|}
\hline Aircraft & $\bar{T}$ & $\left|\epsilon_{\bar{T}}\right|$ \\
\hline Subfleet $A C_{J}$ & 1.424 & 0 \\
Aircraft 1 $A C_{1}$ & 1.464 & 0.040211 \\
Aircraft $2 A C_{2}$ & 1.472 & 0.048477 \\
Aircraft $3 A C_{3}$ & 1.474 & 0.050340 \\
Aircraft 4 $A C_{4}$ & 1.479 & 0.055188 \\
Aircraft $5 A C_{5}$ & 1.483 & 0.058839 \\
\hline
\end{tabular}

Table 1 Average mission duration for fleet and selection of aircraft.

Each mission $m_{n}$ of the fleet as well as for the five aircraft $m_{j}$ individually is categorized with respect to mission duration $T_{n} . T_{n}$ is divided into quarter hour intervals, which results in 20 categories $c_{i}$. This is obtained in a distribution $d_{j}$ of number of flights over mission duration for each aircraft $A C_{j}$. The mission categorization for the fleet $A C_{J}$ is displayed in Fig. 4. For a more detailed analysis of the resulting five aircraft, the mission distribution with respect to the duration is investigated. All missions with a duration between zero and five hours are taken into account. This time span is divided into $0.25 \mathrm{~h}$-long intervals, which results in 20 categories $c_{i}$. The corresponding distribution of all mission of the fleet $d^{A C_{J}}$ is shown in Fig. 4 . 


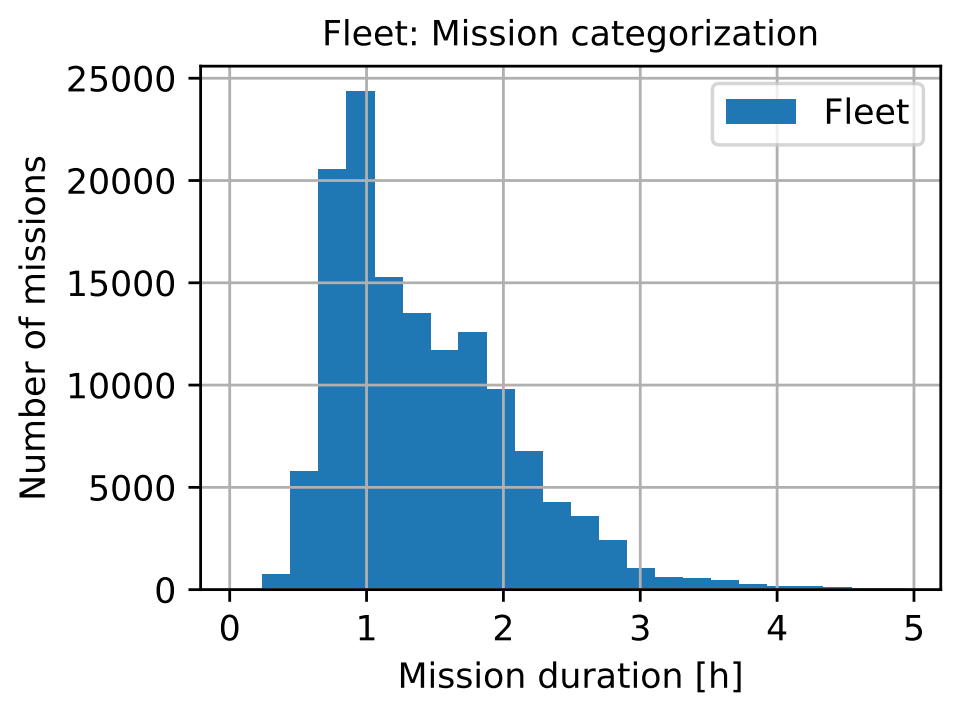

Fig. 4 Fleet mission duration distribution.

To compare the distribution $d^{A C_{j}}$ of a single aircraft with the whole fleet, each of the $d^{A C_{j}}$ is normalized by the total number of missions $N^{d^{A C_{j}}}$ represented by each distribution.

$$
N^{A C_{j}}=\sum_{i=1}^{I} n_{i}^{A C_{j}} \quad \rightarrow \quad \sum_{i=1}^{I} \frac{n_{i}^{A C_{j}}}{N^{A C_{j}}}=1
$$

In Eq. $10 p, i$ is the index of the category. The number of missions in the $i^{\text {th }}$ category is represented by $n_{i} . N^{A C_{j}}$ is the total number of missions of the $j^{\text {th }}$ aircraft. Using the normalization from Eq. 10], the distribution of a single aircraft can be compared to the distribution of the fleet. The comparison of the aircraft distribution $d^{A C_{j}}$ with the fleet distribution $d^{A C_{J}}$ is shown in Fig. 5a for $A C_{1}$, in Fig. $5 \mathrm{~b}$ for $A C_{2}$, in Fig. $5 \mathrm{c}$ for $A C_{3}$, in Fig. $5 \mathrm{~d}$ for $A C_{4}$ and in Fig. $5 \mathrm{e}$ for $A C_{5}$. The difference in every category $c_{i}$ between fleet and individual aircraft is determined by

$$
E^{A C_{j}}=\sum_{i=0}^{I}\left(m_{i}^{A C_{J}}-m_{i}^{A C_{j}}\right)^{2}
$$

The results are summarized in Table 2

\begin{tabular}{|c|c|}
\hline Aircraft $A C_{j}$ & $E^{A C_{j}}$ \\
\hline Aircraft $1 A C_{1}$ & 0.0161 \\
Aircraft $2 A C_{2}$ & 0.0131 \\
Aircraft $3 A C_{3}$ & 0.0141 \\
Aircraft $4 A C_{4}$ & 0.0121 \\
Aircraft $5 A C_{5}$ & 0.0143 \\
\hline
\end{tabular}

Table 2 Quadratic difference in mission distribution between indivudal aircraft $A C_{j}$ and fleet $A C_{J}$.

Based on that $A C_{4}$ is chosen as a representative aircraft for the fleet, because $d^{A C_{4}}$ is closest to $d^{A C_{J}}$ according to $E^{A C_{4}}$. In order to determine representative load time histories for the usage of this aircraft, missions for simulation need to be defined. The missions are defined by further investigating Fig. 5d According to this distribution, for each mission time category $c_{i}^{A C_{j}}$ the flight $f_{i}^{A C_{j}}$ is searched where most missions $m_{i}^{A C_{j}}$ were performed, therefore contributes most to this category. This results in 15 different missions displayed in Fig. 6 These fifteen missions are simulated within the 


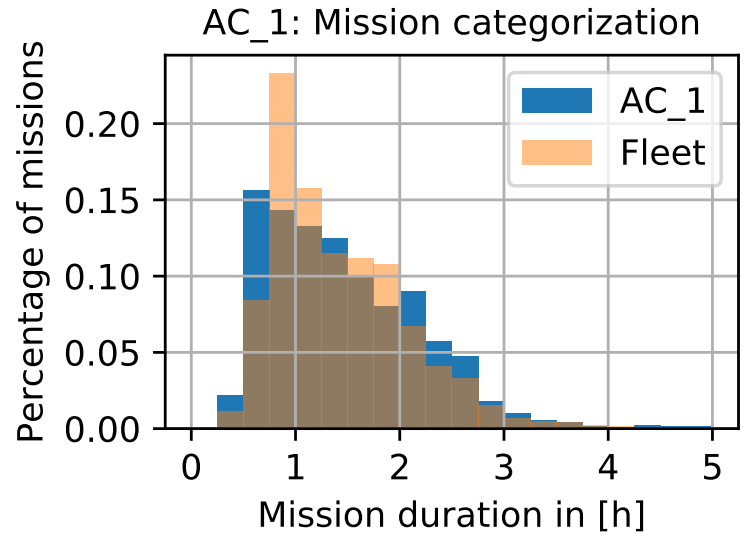

(a) Aircraft 1

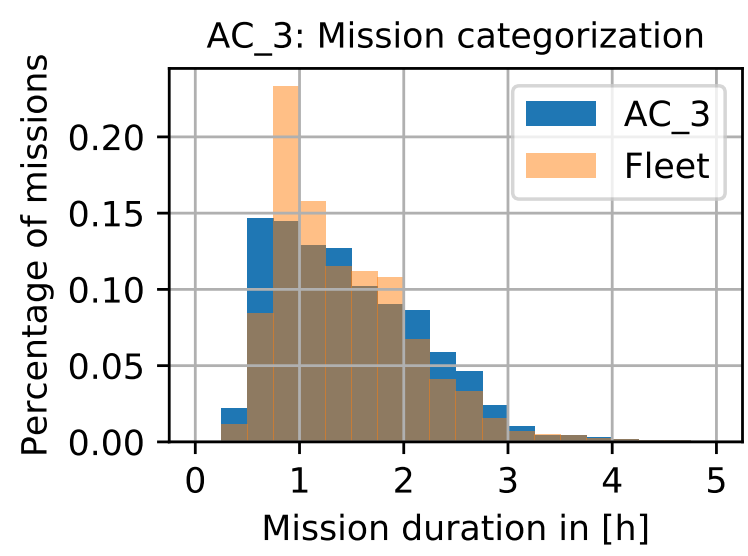

(c) Aircraft 3

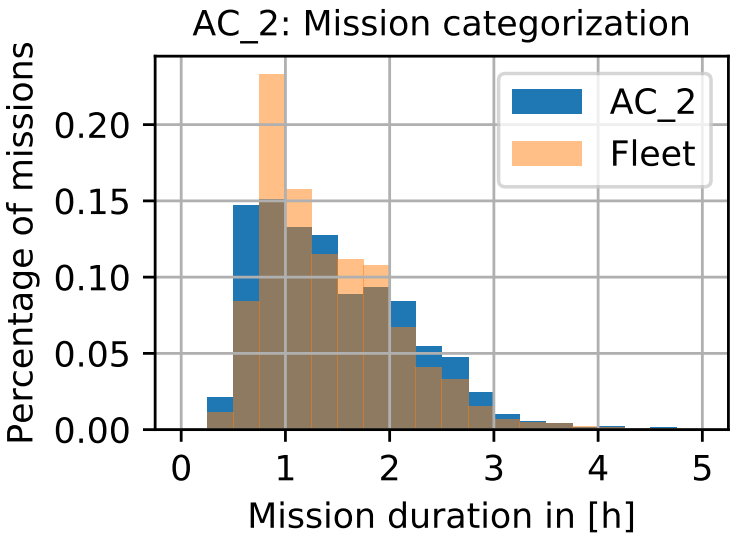

(b) Aircraft 2

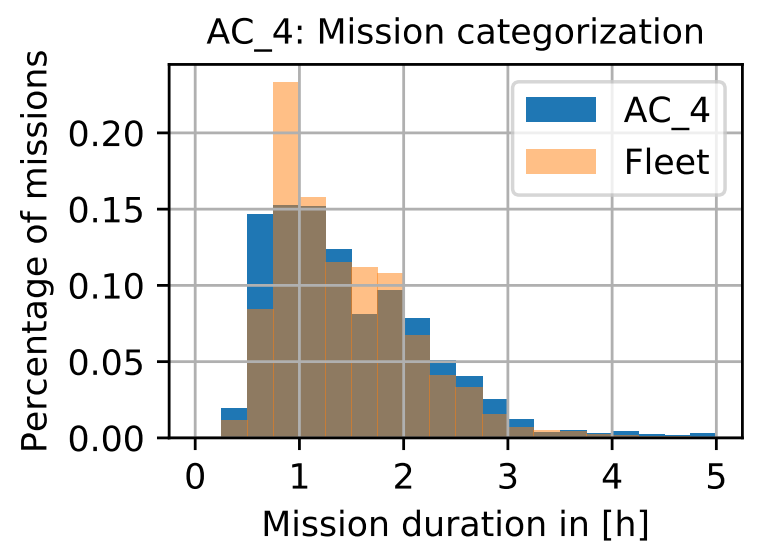

(d) Aircraft 4

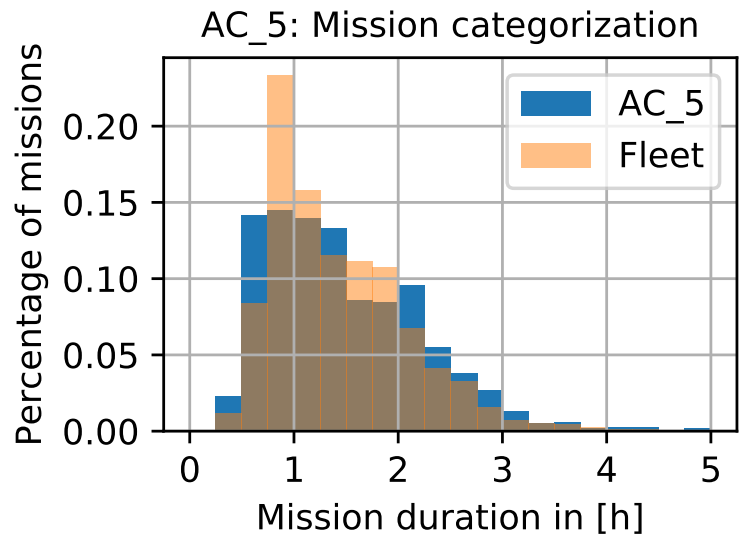

(e) Aircraft 5

Fig. 5 Flight mission duration histograms for five different aircraft, see Eq. 10 .

simulation environment. Exemplary for the whole airframe, the loads are derived for the wing root bending moment of the left wing. The resulting loads during the mission are shown in Fig. 7. Since the missions vary in mission duration, the mission duration is normalized. Furthermore the wingroot bending moment is normalized with respect to the 
average wingroot bending moment of each mission. These results can now be further processed by any algorithm to monitor the impact of the encountered loads on the aircraft structure.

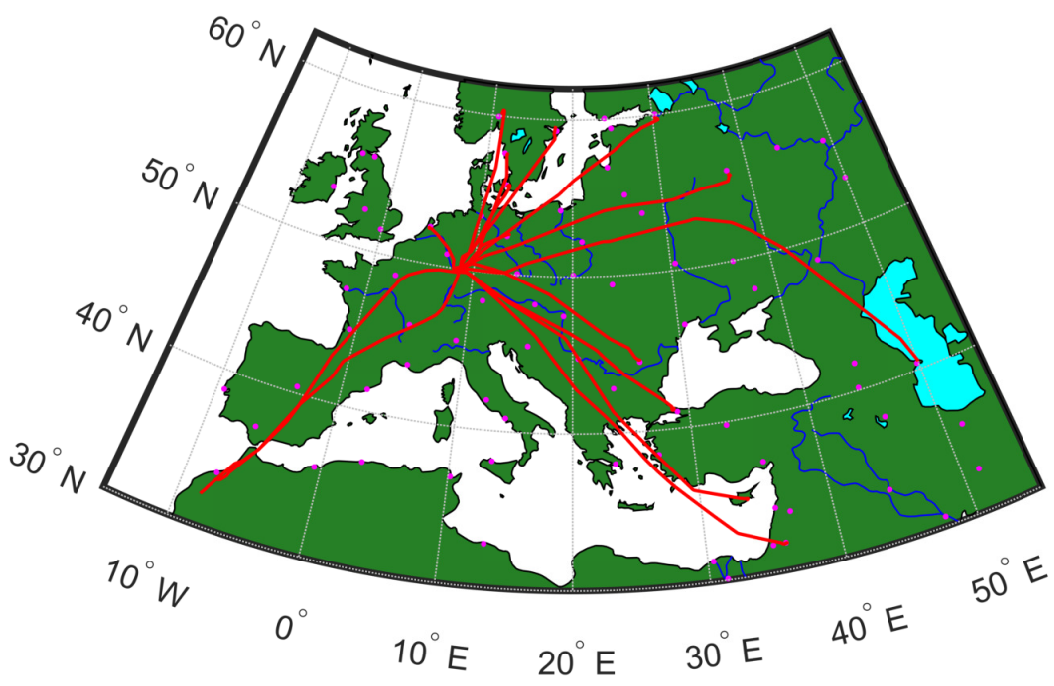

Fig. 6 Representative missions for Aircraft 4.

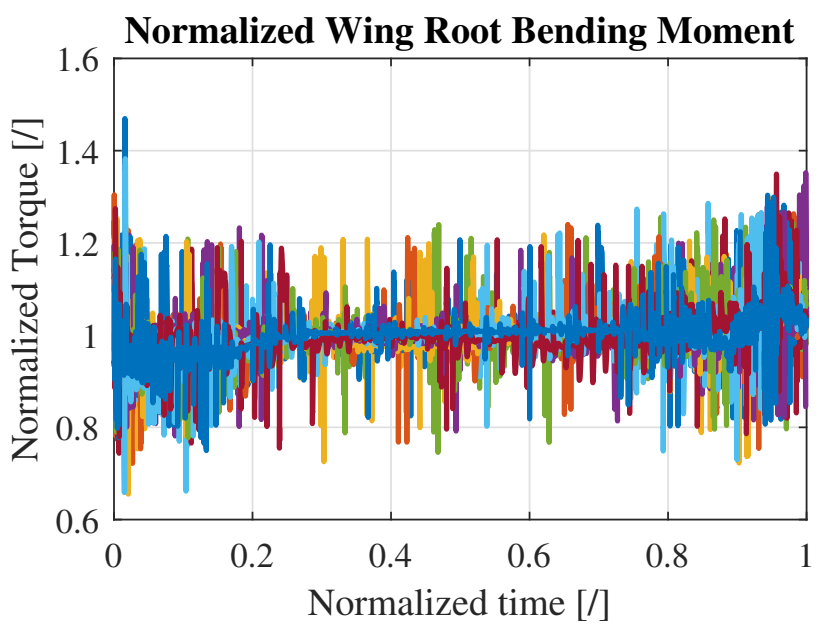

Fig. 7 Normalized Wing Root Bending Moment for representative missions of Aircraft 4.

\section{Conclusions}

In this paper, a framework is proposed which offers, by contrast to the standard loads calculation approach, the possibility to calculate loads for whole flight missions. The functionality is shown for 15 missions, which represent a fleet of 36 aircraft. The loads can be derived at every point of the aircraft structure. As an example, the wing root bending moment is shown. These resulting load-time histories can allow, e.g., deriving stresses within the aircraft structure component of interest. With a subsequent fatigue analysis, a health index can be derived. According to this health index, a maintenance schedule can be derived according to the specific usage of for example this component. In order to make the results of the loads estimation framework more accurate, the following future work will be conducted. The change in aircraft mass due to fuel burn needs to be taken into account since the mass impacts not only the structural modes but also the loads which are required for steering the aircraft. Furthermore, instead of setting up the aerodynamic model according to one specific altitude and Mach-Number, the aerodynamic model needs to be adapted continuously 
during the flight mission according to the flight state and environment.

Currently, only in-flight loads are taken into account, which needs to be extended to ground loads as well. Future work will contain the implementation of loads due to atmospheric disturbances like continuous turbulence and discrete gust.

\section{Acknowledgements}

This work was performed in the framework of the European Union's Horizon 2020 research and innovation programme and is part of the "Process and methods for E2E Maintenance Architecture development and demonstrations and solutions for technology integration" (DEMETER) project with the grant agreement No 685704.

\section{References}

[1] Pusch, M., Knoblach, A., and Kier, T., "Integrated Optimization of Ailerons for Active Gust load alleviation," Proc. of International Forum on Aeroelsticity and Structural Dynamics, Saint Petersburg, Russia, 2015.

[2] Pusch, M., Ossmann, D., and Luspay, T., "Structured Control Design for a Highly Flexible Flutter Demonstrator," Aerospace, Vol. 6, No. 3, 2019, pp. 1-20. doi:10.3390/aerospace6030027.

[3] Ossmann, D., and Poussot-Vassal, C., "Design and Assessment of a Two Degree of Freedom Gust Load Alleviation System," Proc. of 5th CEAS Conference on Guidance, Navigation and Control, 2019.

[4] Wang, Y., Gogu, C., Binaud, N., Bes, C., Haftka, R. T., and Kim, N. H., "A cost driven predictive maintenance policy for structural airframe maintenance," Chinese Journal of Aeronautics, Vol. 30, No. 3, 2017, pp. 1242-1257. doi:10.1016/j.cja.2017.02.005, URL http://wwW.sciencedirect.com/science/article/pii/S1000936117300201

[5] Kier, Thiemo M., and Gertjan Looye, “Unifying Manoeuvre and Gust Loads Analysis Models,”, 2009.

[6] Hofstee, J., Kier, T., Cerulli, C., \& Looye, G, "A variable, fully flexible dynamic response tool for special investigations (VarLoads),", 2003.

[7] Lambregts, A. A., “TECS Generalized Airplane Control System Design - An Update,” Advances in Aerospace Guidance, Navigation and Control, edited by Q. Chu, B. Mulder, D. Choukroun, E.-J. Kampen, C. Visser, and G. Looye, Springer, Berlin and Heidelberg, 2013, pp. 503-534. doi:10.1007/978-3-642-38253-6\{ \textunderscore\}30.

[8] Lambregts, A. A., “THCS Generalized Airplane Control System Design,”, 2013.

[9] Grondman, F., Looye, G., Kuchar, R. O., Chu, Q. P., and van Kampen, E.-J., "Design and Flight Testing of Incremental Nonlinear Dynamic Inversion-based Control Laws for a Passenger Aircraft," 2018 AIAA Guidance, Navigation, and Control Conference, American Institute of Aeronautics and Astronautics, Reston, Virginia, 01082018, p. 1. doi:10.2514/6.2018-0385. 